\title{
Indoor Positioning Using Ultrawideband and Inertial Measurements
}

\author{
Manon Kok, Jeroen D. Hol and Thomas B. Schon
}

Linköping University Post Print

Tweet

N.B.: When citing this work, cite the original article.

Manon Kok, Jeroen D. Hol and Thomas B. Schon, Indoor Positioning Using Ultrawideband and Inertial Measurements, 2015, IEEE Transactions on Vehicular Technology, (64), 4, 1293 1303.

\section{http://dx.doi.org/10.1109/TVT.2015.2396640}

(C2015 IEEE. Personal use of this material is permitted. However, permission to reprint/republish this material for advertising or promotional purposes or for creating new collective works for resale or redistribution to servers or lists, or to reuse any copyrighted component of this work in other works must be obtained from the IEEE.

\section{http://ieeexplore.ieee.org/}

Postprint available at: Linköping University Electronic Press

http://urn.kb.se/resolve?urn=urn:nbn:se:liu:diva-118060 


\title{
Indoor positioning using ultrawideband and inertial measurements
}

\author{
Manon Kok, Jeroen D. Hol, and Thomas B. Schön Senior Member, IEEE
}

\begin{abstract}
In this work we present an approach to combine measurements from inertial sensors (accelerometers and gyroscopes) with time of arrival measurements from an ultrawideband system for indoor positioning. Our algorithm uses a tightlycoupled sensor fusion approach, where we formulate the problem as a maximum a posteriori problem that is solved using an optimization approach. It is shown to lead to accurate $6 \mathrm{D}$ position and orientation estimates when compared to reference data from an independent optical tracking system. To be able to obtain position information from the ultrawideband measurements, it is imperative that accurate estimates of the ultrawideband receivers' positions and their clock offsets are available. Hence, we also present an easy-to-use algorithm to calibrate the ultrawideband system using a maximum likelihood formulation. Throughout this work, the ultrawideband measurements are modeled by a tailored heavy-tailed asymmetric distribution to account for measurement outliers. The heavy-tailed asymmetric distribution works well on experimental data, as shown by analyzing the position estimates obtained using the ultrawideband measurements via a novel multilateration approach.
\end{abstract}

Index Terms-Ultrawideband, inertial sensors, calibration, sensor fusion, heavy-tailed noise distribution.

\section{INTRODUCTION}

$\mathbf{U}$ LTRA-WIDEBAND (UWB) is a relatively new and promising radio technology with applications in for example radar, communication and localization. UWB technology typically makes use of impulse radio with very short pulses. These are typically in the order of $1 \mathrm{~ns}$, opening up for high spatial resolution. This characteristic makes UWB very suitable for localization purposes. It has successfully been applied in a wide variety of localization applications, such as industrial [1], health-care [2], [3] and motion capture [4]. UWB positioning accuracy is reported to be in the order of decimeters [1], [2]. Although UWB systems do not necessarily require line-of-sight visibility [5], the UWB measurements do suffer from multipath and non-line-of-sight (NLOS) conditions, resulting in measurement outliers.

Inertial sensors consist of accelerometers and gyroscopes measuring the acceleration and angular velocity of the sensor. The inertial sensor measurements need to be integrated to obtain position and orientation estimates. These position and

Copyright (c) 2015 IEEE. Personal use of this material is permitted. However, permission to use this material for any other purposes must be obtained from the IEEE by sending a request to pubs-permissions@ieee.org.

M. Kok is with the Department of Electrical Engineering, Linköping University, SE-581 83 Linköping, Sweden e-mail: (see http://users.isy.liu.se/ $\mathrm{en} / \mathrm{rt} / \mathrm{manko} /$ ).

J. D. Hol is with Xsens Technologies B.V., P.O. Box 559, 7500 AN Enschede, the Netherlands e-mail: jeroen.hol@xsens.com.

T. B. Schön is with the Department of Information Technology, Uppsala University, SE-751 05 Uppsala, Sweden e-mail: (see http://user.it.uu.se/ $\sim$ thosc112/,
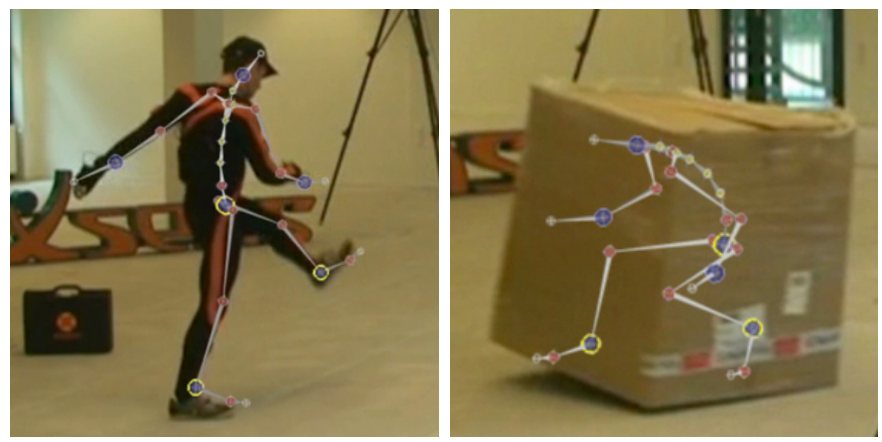

Fig. 1. Example application showing a subject with 17 inertial sensors placed on the body and 3 UWB transmitters placed on the head and on both feet. The pose estimates are visualized by the "skeleton" overlayed in the images. As shown (right), the solution remains valid even in non-line-of-sight conditions.

orientation estimates are accurate on a short time scale, but suffer from integration drift. Inertial sensors have successfully been used to estimate 6D position and orientation (pose) in combination with systems providing position information such as GPS and UWB, see e.g. [6] and the references therein.

In this work we present an indoor positioning approach using inertial sensors and time of arrival (TOA) measurements from an UWB system. We use a setup where a number of UWB receivers are placed in an indoor environment. Our focus is on combining information from an UWB transmitter and an inertial measurement unit (IMU) to estimate the $6 \mathrm{D}$ pose of the IMU. The IMU and the transmitter are assumed to be rigidly attached to each other. Our approach can be extended to for example estimate the 6D pose of a human body where a subject wearing multiple IMUs and multiple UWB transmitters walks through the UWB measurement volume, as shown in Fig. 1] This work builds on [6], [7], where an extended Kalman filter (EKF) was used in combining the inertial and UWB measurements to estimate the $6 \mathrm{D}$ pose of the sensor. Outlier rejection was used to remove UWB measurements affected by multipath and NLOS. In this work we instead solve the problem using an optimization-based approach similar to the approach used in [8]. Using this approach, it is possible to assume more general measurement distributions. Hence, we model the UWB measurements using a heavy-tailed asymmetric distribution which is specifically tailored for this particular application. This distribution naturally handles the possibility of measurement delays due to multipath and NLOS while not allowing for the possibility of measurements arriving earlier, i.e. traveling faster than the speed of light. We will show that accurate position and orientation estimates are obtained by comparing our results to those obtained from an independent 
optical reference system.

To be able to obtain position information from the UWB measurements, the positions of the receivers must be known and the receiver clocks have to be synchronized. To avoid the typically labor-intensive and time-consuming process of manually surveying the receiver positions, we present an easy-to-use calibration method that automates this process. Our previous solution presented in [9] assumed "clean" measurements, i.e. it was assumed that no outliers were present. In this work, we will instead solve the calibration problem modeling the UWB measurements using the heavy-tailed asymmetric distribution mentioned earlier to naturally handle the measurement outliers.

To experimentally validate the UWB model using the heavytailed asymmetric distribution, we will use the UWB measurements in a novel multilateration approach to determine the position of a mobile transmitter. Here, we assume that the UWB system has previously been calibrated using our calibration algorithm. We will show that the position estimates obtained using the heavy-tailed asymmetric distribution are considerably better than the ones obtained using either a Gaussian distribution or a heavy-tailed symmetric Cauchy distribution.

In Section III we provide more background to our work by relating it to previous work in the area. In Section III we clearly formulate the problem. The sensors and their corresponding measurement models are introduced in Section IV The UWB calibration problem is subsequently solved in Section $V$ The solution to the sensor fusion problem, where we also make use of the inertial measurements can be found in Section VI. The experimental results and the conclusions are then provided in Section VII and Section VIII respectively.

\section{RELATED WORK}

In this work we make use of TOA measurements from an UWB system. Our UWB setup consists of a network of synchronized and stationary (rigidly fixed, mounted) receivers, all acquiring very precise TOA measurements of signals originating from a mobile transmitter. The low-cost transmitters in our setup have an inaccurate clock and can hence not provide accurate information concerning the time of transmission. For a general introduction to UWB technology and its use in positioning applications, see e.g. [3], [10].

The process of determining the transmitter position from TOA measurements is referred to as trilateration or, more accurately, multilateration. It is a well-studied topic and many algorithms have been reported in the literature, see e.g. [3], $[10]-[12]$. A common multilateration technique is to eliminate the time of transmission by constructing time difference of arrival (TDOA) measurements from pairs of TOA measurements. The resulting set of hyperbolic equations can then be solved for position [13]. The drawback of this approach is that the constructed TDOA measurements are no longer independently distributed which complicates the calculations. In this work we use a well-known equivalent approach where we instead model the time of transmission as an unknown quantity.

Ideally, the UWB signal travels directly from the transmitter to the different receivers. In that case, the TOA measurements are directly related to the distance traveled. In case the signal encounters a medium which delays or reflects the signal, however, the time of flight is prolonged and the pulse will be delayed. This can result in large estimation errors when assuming that the UWB measurements are Gaussian distributed, as will be illustrated in Section VII-A. The problem of how to robustly deal with outliers in the measurements has received a lot of attention, see e.g. [14] for a good survey containing relevant entry-points into the literature on this topic commonly referred to as robust statistics. A common approach is to model the outliers in terms of the probability of NLOS and introduce a delay represented by a heavy-tailed positive-mean probability density function (PDF) such as a shifted Gaussian or an exponential, see e.g. [15], [16]. Based on [15], the localization approach presented in [17] builds spatial models representing the probability of NLOS in a certain area. In our approach we do not specifically model the probability of the number of outliers. Instead, we model the UWB measurements using a specifically tailored asymmetric heavy-tailed noise distribution. By estimating the width of this distribution, the algorithm can automatically adapt the width to the specific measurement data. The use of this distribution can straightforwardly be incorporated in any maximum likelihood (ML) or maximum a posteriori (MAP) estimation algorithm, without including any additional parameters.

UWB approaches typically assume that the receiver positions are known and that their clocks are synchronized, explaining why there are relatively few UWB calibration algorithms available in the literature, see e.g. [6], [7]. However, ideas for UWB calibration can be obtained from the wide range of literature on sensor localization, see for instance [18]. The challenge again comes down to the possibility of measurement errors due to NLOS and/or multipath, resulting in a reduced quality of the calibration results. Hence, we assume a tailored asymmetric heavy-tailed distribution in our calibration algorithm to represent these errors.

When combining inertial measurements with UWB measurements, a tightly-coupled or a loosely-coupled approach can be used. In a loosely-coupled approach, the UWB measurements are used to obtain position estimates using a multilateration approach. These position estimates are subsequently used as artificial position measurements in the sensor fusion approach, see e.g. [19]-[21]. A tightly-coupled approach instead makes direct use of each individual TOA measurement, see for instance [7], [22]. An advantage of a tightly-coupled approach is that it does not suffer from the loss of information that typically arises from pre-processing of the UWB measurements that has to be performed in a loosely-coupled approach. This is mainly due to approximations of statistical distributions, but in extreme cases measurements are also ignored, for instance when there are not enough TOA measurements for multilateration. By instead making direct use of the sensor measurements, we can make maximal use of the available information. The advantage of a tightly-coupled approach is experimentally shown for the case of UWB measurements in for instance [23].

One way to solve the sensor fusion problem is then to use an EKF. To allow for the presence of delayed measurements, 
approaches based on outlier rejection [6], [7] and robust EKF formulations [24] have previously been used. In this work we will instead formulate a tightly-coupled approach by formulating and solving an optimization problem. This also straightforwardly opens up for exploiting non-Gaussian distributions and for estimating additional parameters.

\section{PROBLEM FORMULATION}

To determine an IMU's 6D position and orientation, its measurements are combined with those originating from an UWB transmitter that is rigidly attached to the sensor. The UWB transmitter sends pulses to a number of stationary UWB receivers as illustrated in Fig. 2. The receivers measure the times of arrival of the pulses at their different locations. Provided that the receiver positions are known and that their clocks are synchronized, the position of the transmitter can be inferred from these measurements. Although the receivers are synchronized to a central clock, they each have a small, constant clock offset due to for instance differences in cable lengths. The receiver positions and clock offsets are computed using our calibration algorithm.

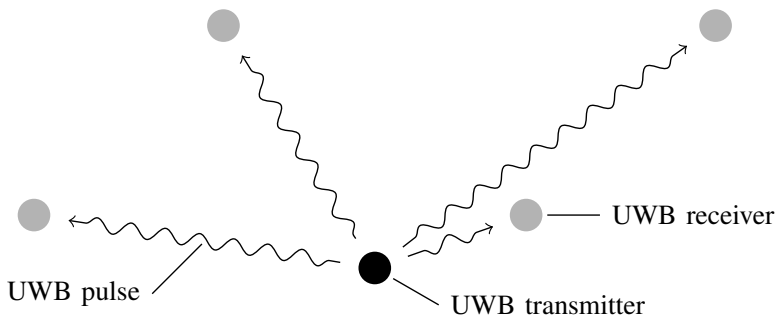

Fig. 2. The UWB setup consists of a number of stationary receivers acquiring TOA measurements of signal pulses originating from a mobile transmitter.

Let us denote the model parameters estimated by the calibration algorithm by $\theta$. The calibration algorithm computes an ML estimate $\widehat{\theta}^{\mathrm{ML}}$. For a setup with $m=1, \ldots, M$ receivers and $l=1, \ldots, L$ transmitters, the UWB measurements are denoted $y_{\mathrm{u}, m l k}$ for $k=1, \ldots, K$ UWB pulses. Note that the absolute receiver positions and clock offsets are neither observable, nor relevant. We are interested in estimating the receivers' relative positions and clock offsets. Hence, an arbitrary choice of an UWB coordinate frame and a reference clock offset are used as constraints in the ML problem. The problem of calibrating the UWB setup is therefore formulated as a constrained ML problem according to

$$
\begin{aligned}
\max _{\theta \in \Theta} & \prod_{k=1}^{K} \prod_{l=1}^{L} \prod_{m=1}^{M} p_{\theta}\left(y_{\mathrm{u}, m l k}\right), \\
\text { s.t. } & A \theta=b,
\end{aligned}
$$

where $p_{\theta}(y)$ denotes the PDF of $y$ parametrized by $\theta$. The matrix $A$ and the vector $b$ are used to describe the linear constraints on the parameter vector $\theta$, due to the choice of the coordinate frame and the reference clock offset. The calibration problem (1) is solved in Section V] where we also provide the resulting calibration algorithm. In that section, the constraints in (1) are also defined more explicitly.
When the UWB system has been calibrated, the UWB measurements can be combined with inertial measurements to determine the $6 \mathrm{D}$ pose of the sensor. Hence, we estimate the state vector $x_{1: N}$ which contains the position of the sensor, its orientation and additional information. Denoting the accelerometer measurements by $y_{\mathrm{a}, t}$ for $t=1, \ldots, N$, the gyroscope measurements by $y_{\omega, t}$ and the UWB measurements by $y_{\mathrm{u}, m k}$ for $k=1, \ldots, K$ pulses and $m=1, \ldots, M$ receivers, the state is computed by solving the following MAP problem,

$$
\max _{x_{1: N}} p\left(x_{1: N} \mid\left\{y_{\mathrm{a}, t}, y_{\omega, t}\right\}_{t=1}^{N},\left\{\left\{y_{\mathrm{u}, m k}\right\}_{m=1}^{M}\right\}_{k=1}^{K}\right) .
$$

Here, $p\left(x_{1: N} \mid y_{1: N}\right)$ denotes the conditional PDF of the state vector $x_{1: N}$ given the measurements $y_{1: N}$. The subscript $k$ used for the UWB measurements indicates that the UWB measurements do not necessarily have the same sampling frequency as the inertial measurements. The solution to the sensor fusion problem (2) is provided in Section VI, together with the resulting algorithm.

\section{SENSOR MODELS}

Our UWB system consists of a network of stationary receivers which can track a large number of small, batterypowered inexpensive transmitters [2]. A transmitter and a receiver are shown in Fig. 3 . In our sensor fusion approach, we combine UWB measurements with inertial measurements. The IMU is shown in Fig. 4

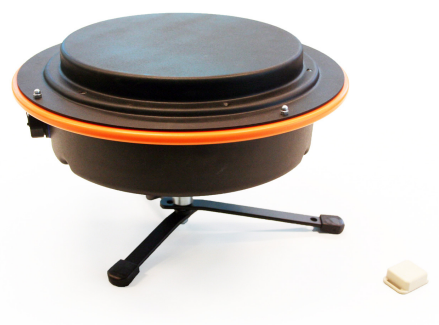

Fig. 3. Hardware used in an UWB setup. More specifically, an UWB receiver and a small, battery-powered UWB transmitter.

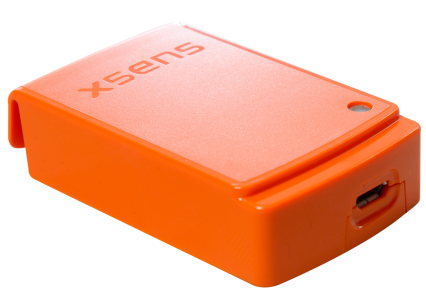

Fig. 4. An IMU containing a 3-axis accelerometer and a 3-axis gyroscope.

In this section we will introduce our sensor models, starting with the UWB measurement model in Section IV-A. Subsequently, the inertial measurement models will be introduced in Section IV-B. 


\section{A. Modeling the ultrawideband measurements}

For the UWB setup with $m=1, \ldots, M$ receivers and $l=$ $1, \ldots, L$ transmitters, the TOA measurement $y_{\mathfrak{u}, m l k}$ of receiver $m$, originating from transmitter $l$ and pulse $k$, is modeled as

$$
y_{\mathrm{u}, m l k}=\tau_{l k}+\frac{1}{c}\left\|r_{m}^{\mathrm{n}}-t_{l k}^{\mathrm{n}}\right\|_{2}+\Delta \tau_{m}+e_{\mathrm{u}, m l k} .
$$

Here, $c$ denotes the speed of light, $\tau_{l k}$ is the time of transmission of pulse $k$ from transmitter $l, t_{l k}^{\mathrm{n}}$ is the position of transmitter $l$ at the time of transmitting the $k^{\text {th }}$ pulse, $r_{m}^{\mathrm{n}}$ is the position of the $m^{\text {th }}$ receiver and $\Delta \tau_{m}$ is its clock-offset. The superscript $n$ denotes the navigation frame. It is a local coordinate frame that is aligned with the earth's gravity and with the axes of the frame defined during the UWB calibration, as already discussed in Section III.

Due to NLOS conditions and/or multipath we expect a small number of measurements to be delayed. Hence, it does not make sense to model $e_{\mathrm{u}, m l k}$ using a Gaussian distribution. In [6], a new multilateration approach was presented, where the possibility of delayed measurements was modeled by including a positive parameter $\delta_{\mathrm{u}, m k}$ explicitly representing the delay of pulse $k$ to receiver $m$ in the measurement equation (3). The parameters $\delta_{\mathrm{u}, m k}$ were assumed to have an exponential prior. Hence, [6] models the delay of each pulse to each receiver as a parameter to be estimated. This was shown to lead to accurate position estimates, but it also introduced $M$ additional model parameters for each pulse $k$.

In this work, we omit the parameters $\delta_{\mathfrak{u}, m k}$ and instead model the possibility of delays in terms of the distribution of the noise $e_{\mathrm{u}, m l k}$. We assume an asymmetric distribution where a heavy-tailed Cauchy distribution allows for measurement delays while a Gaussian distribution excludes the physically unreasonable possibility of pulses traveling faster than the speed of light as

$$
e_{\mathbf{u}, m l k} \sim \begin{cases}(2-\alpha) \mathcal{N}\left(0, \sigma^{2}\right) & \text { for } e_{\mathbf{u}, m l k}<0 \\ \alpha \text { Cauchy }(0, \gamma) & \text { for } e_{\mathbf{u}, m l k} \geq 0\end{cases}
$$

The presence of the constants $\alpha$ and $2-\alpha$ is motivated by the fact that the proposed asymmetric PDF needs to integrate to one and hence

$$
\begin{aligned}
& \int_{-\infty}^{0}(2-\alpha) \frac{1}{\sqrt{2 \pi \sigma^{2}}} \exp \left(-\frac{e_{\mathrm{u}, m l k}^{2}}{2 \sigma^{2}}\right) \mathrm{d} e_{\mathrm{u}, m l k},+ \\
& \int_{0}^{\infty} \alpha \frac{1}{\pi \gamma}\left(\frac{1}{1+\frac{e_{\mathrm{u}, m l k}^{2}}{\gamma^{2}}}\right) \mathrm{d} e_{\mathrm{u}, m l k}=\frac{1}{2}(2-\alpha)+\frac{1}{2} \alpha=1,
\end{aligned}
$$

where we have made use of the fact that the Gaussian and Cauchy PDFs integrate to one and are symmetric. Imposing the constraint that the distribution is continuous at $e_{\mathrm{u}, m l k}=0$ allows us to express $\alpha$ in terms of $\sigma$ and $\gamma$ according to

$$
\frac{\alpha}{\pi \gamma}=\frac{2-\alpha}{\sqrt{2 \pi \sigma^{2}}} \Leftrightarrow \alpha=\frac{2 \pi \gamma}{\sqrt{2 \pi \sigma^{2}}+\pi \gamma} .
$$

The proposed asymmetric PDF and its corresponding negative log-likelihood, given by

$$
-\log p\left(e_{\mathrm{u}, m l k}\right)= \begin{cases}\mathcal{L}_{G} & \text { for } e_{\mathrm{u}, m l k}<0, \\ \mathcal{L}_{C} & \text { for } e_{\mathrm{u}, m l k} \geq 0,\end{cases}
$$
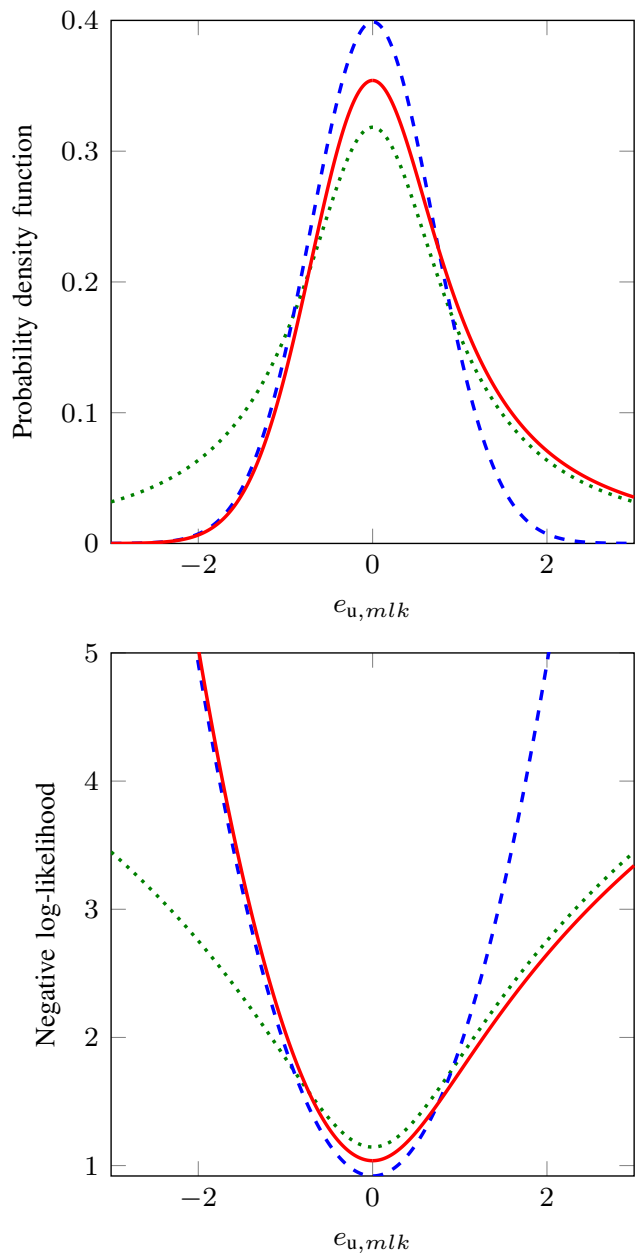

Fig. 5. PDF (top) and negative log-likelihood (bottom) of a $\mathcal{N}(0,1)$ distribution (blue, dashed), a Cauchy $(0,1)$ distribution (green, dotted) and the asymmetric distribution (4) asssuming $\sigma=\gamma=1$ and $\alpha$ according to 6 (red).

$$
\begin{aligned}
& \mathcal{L}_{G} \triangleq \frac{e_{u, m l k}^{2}}{2 \sigma^{2}}+\frac{1}{2} \log \sigma^{2}+\frac{1}{2} \log 2 \pi-\log (2-\alpha), \\
& \mathcal{L}_{C} \triangleq \log \left(1+\frac{e_{u, m l k}^{2}}{\gamma^{2}}\right)+\frac{1}{2} \log \gamma^{2}+\log \pi-\log \alpha .
\end{aligned}
$$

are both depicted in Fig. 5 in red. For comparison, the Gaussian and Cauchy PDFs are also depicted, in blue and green, respectively.

From the experimental results in Section VII-A it will be shown that exploiting the asymmetry of the actual noise distribution is especially helpful in the presence of a large number of outliers.

\section{B. Modeling the inertial measurements}

An IMU containing a 3-axis accelerometer and a 3-axis gyroscope was shown in Fig. 4. The inertial measurements are resolved in the body frame $b$. Its origin lies in the center of the accelerometer triad and its axes are aligned with the casing. The gyroscope measures the sensor's angular velocity $\omega_{t}$. Its measurements $y_{\omega, t}$ are modeled as

$$
y_{\omega, t}=\omega_{t}+\delta_{\omega}+e_{\omega, t}
$$


where $\delta_{\omega}$ denotes the gyroscope bias and $e_{\omega, t} \sim \mathcal{N}\left(0, \Sigma_{\omega}\right)$. We assume $\Sigma_{\omega}=\sigma_{\omega}^{2} \mathcal{I}_{3}$ where $\mathcal{I}_{3}$ denotes the $3 \times 3$ identity matrix. The accelerometer measures the external specific force $f_{t}^{\mathrm{b}}$ exerted on the sensor. It consists of the sensor's linear acceleration $a_{t}^{\mathrm{n}}$ and the gravity vector $g^{\mathrm{n}}$, both resolved in the navigation frame $n$. The accelerometer measurements $y_{\mathrm{a}, t}$ are modeled as

$$
y_{\mathrm{a}, t}=f_{t}^{\mathrm{b}}+\delta_{\mathrm{a}}+e_{\mathrm{a}, t}=R_{t}^{\mathrm{bn}}\left(a_{t}^{\mathrm{n}}-g^{\mathrm{n}}\right)+\delta_{\mathrm{a}}+e_{\mathrm{a}, t},
$$

where $\delta_{\mathrm{a}}$ denotes the accelerometer bias and $e_{\mathrm{a}, t} \sim \mathcal{N}\left(0, \Sigma_{\mathrm{a}}\right)$. We assume $\Sigma_{\mathrm{a}}=\sigma_{\mathrm{a}}^{2} \mathcal{I}_{3}$. The rotation matrix $R_{t}^{\mathrm{bn}}$ represents the rotation from the navigation frame $n$ to the body frame $b$ at time $t$.

Both the gyroscope and the accelerometer biases $\delta_{\omega}$ and $\delta_{\mathrm{a}}$ are slowly time-varying, but we will treat them as constants motivated by the short experimental times used in this work. For longer experiments, $\delta_{\omega}$ and $\delta_{\mathrm{a}}$ can be assumed to be timevarying instead.

The inertial measurements provide information about the position and orientation of the sensor. Integration of the angular velocity measured by the gyroscope leads to information about the sensor's change in orientation. Subtracting gravity from the specific force measured by the accelerometer and double integrating the resulting signal leads to information about the sensor's change in position. The process of estimating position and orientation from the inertial measurements is schematically illustrated in Fig. 6

\section{ULTRAWIDEBAND CALIBRATION}

In this section, we will derive a calibration algorithm to determine the positions $\left\{r_{m}^{\mathrm{n}}\right\}_{m=1}^{M}$ and the clock offsets $\left\{\Delta \tau_{m}\right\}_{m=1}^{M}$ of the receivers using the ML formulation (1). The algorithm makes use of data obtained by moving a single transmitter through the measurement volume. The data collected in this way is denoted $\mathcal{D}_{1}$. Since we do not place the transmitter at known positions, but instead move it around freely, the calibration algorithm aims at simultaneously localizing both the moving transmitter and the receivers. The transmission times of the different pulses $\left\{\tau_{k}^{\mathrm{n}}\right\}_{k=1}^{K}$ are also considered unknown. Note that we have omitted the subscript $l$ since we consider the case of using a single transmitter.

The UWB measurements are modeled according to (3), where the noise $e_{\mathrm{u}, m k}$ is assumed to be distributed according to the asymmetric distribution (4). The parameters $\sigma$ and $\gamma$ are considered unknown and to be estimated, i.e. the algorithm tunes itself and does not rely on a priori knowledge about the accuracy of the UWB measurements. The resulting parameter vector is

$$
\theta=\left(\left\{t_{k}^{\mathrm{n}}, \tau_{k}\right\}_{k=1}^{K},\left\{r_{m}^{\mathrm{n}}, \Delta \tau_{m}\right\}_{m=1}^{M}, \sigma, \gamma\right) .
$$

To make use of the measurement model (4) within our calibration problem (1), we need the following relationship

$$
p_{\theta}\left(y_{\mathfrak{u}, m k}\right)=p_{e_{\mathrm{u}, m k}}\left(y_{\mathrm{u}, m k}-\tau_{k}-\frac{1}{c}\left\|r_{m}^{\mathrm{n}}-t_{k}^{\mathrm{n}}\right\|_{2}-\Delta \tau_{m}\right),
$$

where $\theta$ is defined in $(10)$.

The calibration problem is non-convex and hence needs proper initialization. In Sections $\mathrm{V}-\mathrm{A}$ and $\mathrm{V}-\mathrm{B}$, we introduce a two-step procedure to compute such an initial estimate. In a first step, we obtain an initial estimate of the receiver positions $\left\{r_{m, 0}^{\mathrm{n}}\right\}_{m=1}^{M}$ and their clock offsets $\left\{\Delta \tau_{m, 0}\right\}_{m=1}^{M}$ using a second data set $\mathcal{D}_{2}$ for which the transmitter positions are known. In a second step, initial estimates of the transmitter positions $\left\{t_{k, 0}^{\mathrm{n}}\right\}_{k=1}^{K}$ and the transmission times $\left\{\tau_{k, 0}\right\}_{k=1}^{K}$ are obtained by assuming that the receiver positions and clock offsets are known. This is done using a novel multilateration approach in which the UWB measurements are assumed to be distributed according to the asymmetric heavy-tailed distribution (4) with unknown $\sigma$ and $\gamma$. In Section $\mathrm{V}-\mathrm{C}$ we will then introduce the resulting calibration algorithm which is used to compute an ML estimate of all the unknown parameters $\theta$ defined in $(10)$.

\section{A. Initial estimate: step I}

As a first step of the initial estimation, a second data set, denoted $\mathcal{D}_{2}$, is used to determine the positions and the clock offsets of the receivers. This data is collected by placing a number of UWB transmitters at known locations. To avoid manual measuring of the positions of the transmitters, they are rigidly attached to the receivers. Hence, the relative position of each transmitter with respect to the corresponding receiver is approximately known and constant. This relative distance is denoted $d_{\mathrm{rt}}^{\mathrm{n}}$. As discussed in Section III an arbitrary choice of the reference clock offset and the UWB coordinate frame needs to be used as a constraint to the calibration problem. Hence, the optimization problem can be written as

$$
\begin{aligned}
\widehat{\theta_{1}=\underset{\theta_{1}}{\arg \min }}- & \sum_{m=1}^{M} \sum_{l=1}^{L} \sum_{k=1}^{K} \log p_{\theta_{1}}\left(y_{\mathrm{u}, m l k}\right), \\
\text { s.t. } \quad & A\left(\begin{array}{c}
\operatorname{vec}\left(r_{1: M}^{\mathrm{n}}\right) \\
\left.\Delta \tau_{1: M}\right)
\end{array}\right)=b, \\
& r_{m}^{\mathrm{n}}-t_{m}^{\mathrm{n}}=d_{\mathrm{rt}}^{\mathrm{n}}, \quad m=1, \ldots, M,
\end{aligned}
$$

where the UWB measurements $y_{\mathrm{u}, m l k}$ are modeled according to (3). Since it is not necessary to walk through the measurement volume during the collection of this data, the measurements in the data set $\mathcal{D}_{2}$ can typically be assumed to have little problems with outliers. Hence, $e_{\mathrm{u}, m l k}$ in (3) can fairly accurately be modeled using a Gaussian PDF. Note that this is the only instance in this work where we assume that the UWB measurements are distributed according to a Gaussian. By assuming that the standard deviation of this Gaussian is the same for all $m, l$ and $k$, we have reduced (12) to a constrained least-squares problem. The parameter vector $\theta_{1}$ is given by

$$
\theta_{1}=\left(\left\{r_{m}^{\mathrm{n}}, \Delta \tau_{m}\right\}_{m=1}^{M},\left\{t_{l, \mathcal{D}_{2}}^{\mathrm{n}},\left\{\tau_{l k, \mathcal{D}_{2}}^{\mathrm{n}}\right\}_{k=1}^{K}\right\}_{l=1}^{L}\right) .
$$

The subscript $\mathcal{D}_{2}$ on the (stationary) transmitter positions $t_{l}^{\mathrm{n}}$ and the transmission times $\tau_{l k}^{\mathrm{n}}$ is added to stress that these parameters are only relevant for the dataset $\mathcal{D}_{2}$.

The constraints (12b) are defined as

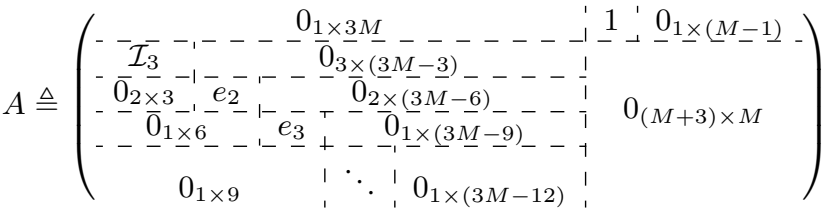

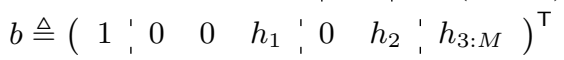




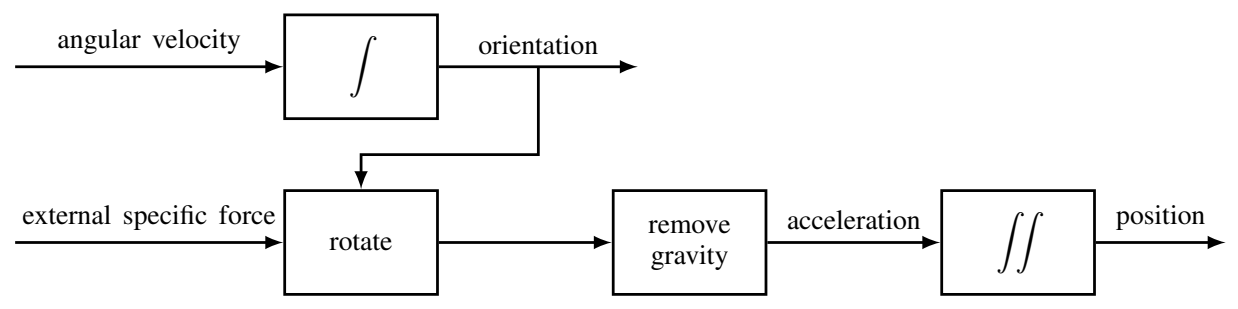

Fig. 6. Schematic illustration of the process of determining position and orientation from inertial measurements, assuming a known initial position, velocity and orientation.

where $e_{2}=\left(\begin{array}{ll}0_{2 \times 1} & \mathcal{I}_{2}\end{array}\right)$ and $e_{3}=\left(\begin{array}{lll}0 & 0 & 1\end{array}\right)^{\top}$. The first row in the matrix $A$ is used to define the reference clock offset. Note that the choice of which receiver to use for this is arbitrary. The remaining rows are used to define the UWB coordinate frame. The first receiver is used to define the origin. The second receiver is used to define the x-axis. The height of each receiver $m=1, \ldots, M$ is constrained to be equal to its measured height $h_{m}$. This ensures that the UWB coordinate frame is gravity-aligned which is beneficial for our sensor fusion approach in Section VI.

The constraint $[12 \mathrm{c}$ ) is used to incorporate the knowledge of the location of the transmitters with respect to the receivers. Note that we assume that each receiver has a transmitter attached to it, i.e. we have $M$ constraints (12c).

The problem (12) is again a non-convex optimization problem and therefore requires a reasonably good starting point. Hence, we start the solver for (12) in a user-specified initial receiver configuration, a noisy, rotated and scaled estimate of the set of receiver positions.

\section{B. Initial estimate: step II - multilateration}

As a second step of the initialization, an initial estimate of the transmitter positions $\left\{t_{k, 0}^{\mathrm{n}}\right\}_{k=1}^{K}$ and the transmission times $\left\{\tau_{k, 0}\right\}_{k=1}^{K}$ is determined for the calibration data set $\mathcal{D}_{1}$ in which a transmitter is moved around in the UWB measurement volume. This problem is solved using a novel multilateration approach, which can also be used stand-alone as will be done in Section VII-A. We model the UWB measurements using the asymmetric heavy-tailed distribution (4). To avoid ad hoc assumptions on $\sigma$ and $\gamma$, we treat them as parameters in an ML problem where we estimate the parameters $\theta_{2}$ with

$$
\theta_{2}=\left(\left\{t_{k}^{\mathrm{n}}, \tau_{k}\right\}_{k=1}^{K}, \sigma, \gamma\right) .
$$

Using the fact that the logarithm is a monotonic function, the resulting optimization problem is given by

$$
\widehat{\theta}_{2}=\underset{\theta_{2}}{\arg \min }-\sum_{k=1}^{N} \sum_{m=1}^{M} \log p_{\theta_{2}}\left(y_{\mathbf{u}, m k}\right),
$$

where the the UWB measurements are modeled as (3) and their noise $e_{\mathrm{u}, m k}$ is given by the asymmetric noise distribution (4). Hence, instead of solving $N$ individual multilateration problems, we solve one optimization problem to determine the transmitter positions, the transmission times as well as the parameters $\sigma$ and $\gamma$.
The multilateration problem formulated in $(16)$ can be solved ${ }^{1}$ using standard Gauss-Newton solvers [25], [26] where the negative log-likelihood, its gradient and approximate Hessian are evaluated at the current iterate. Evaluating (3), the sign of $e_{\mathrm{u}, m k}$ for each pulse $k=1, \ldots, N$ and each receiver $m=1, \ldots, M$ can be used to determine whether the Gaussian or Cauchy negative log-likelihood in (7) should be used.

\section{Resulting calibration algorithm}

The resulting calibration algorithm uses the data set $\mathcal{D}_{1}$ in which a transmitter is moved around in the measurement volume. To obtain an ML estimate of the parameter vector $[10)$, the following constrained optimization problem is solved

$$
\begin{aligned}
\widehat{\theta}^{\mathrm{ML}}=\underset{\theta}{\arg \min } & -\sum_{m=1}^{M} \sum_{k=1}^{K} \log p_{\theta}\left(y_{\mathrm{u}, m k}\right), \\
\text { s.t. } & A\left(\begin{array}{c}
\operatorname{vec}\left(r_{1: M}^{\mathrm{n}}\right) \\
\Delta \tau_{1: M}
\end{array}\right)=b,
\end{aligned}
$$

where we make use of the UWB measurement model (3) and the asymmetric noise distribution (4). The constraints (17b) have already been defined in (14). The problem can be solved using standard constrained Gauss-Newton solvers [25], [26]. The calibration algorithm is summarized in Algorithm 1 .

\footnotetext{
Algorithm 1 Ultrawideband calibration

1: Construct a setup consisting of $M$ stationary receivers.

2: Place $M$ transmitters in close proximity to the receiver antennas and collect a data set $\mathcal{D}_{2}$.

3: Solve (12) using the data set $\mathcal{D}_{2}$ to obtain $\left\{r_{m, 0}^{\mathrm{n}}, \Delta \tau_{m, 0}\right\}_{m=1}^{M}$. The optimization is initialized using a noisy, scaled and rotated estimate of the set of receiver positions provided by the user.

4: Collect a data set $\mathcal{D}_{1}$ by moving a single transmitter throughout the measurement volume.

5: Solve the multilateration problem (16) using the data set $\mathcal{D}_{1}$ with the calibration values of Step 3 to obtain $\left(\left\{t_{k, 0}^{\mathrm{n}}, \tau_{k, 0}\right\}_{k=1}^{K}, \sigma_{0}, \gamma_{0}\right)$.

6: Solve 17 for $\mathcal{D}_{1}$. The optimization is started in

$$
\theta_{0}=\left(\left\{t_{k, 0}^{\mathrm{n}}, \tau_{k, 0}\right\}_{k=1}^{K},\left\{r_{m, 0}^{\mathrm{n}}, \Delta \tau_{m, 0}\right\}_{m=1}^{M}, \sigma_{0}, \gamma_{0}\right),
$$

using the results from Steps 3 and 5

${ }^{1}$ As for any nonlinear optimization problem, good initial estimates help for convergence. Hence, we first estimate a part of the parameter vector (15), choosing $\sigma=\gamma=1$. The resulting parameters are then used to determine a first estimate of $\sigma$ and $\gamma$. Finally, the obtained parameter values are used as initial values for the final optimization.
} 


\section{SENSOR FUSION}

In this section we describe our approach to combine UWB measurements with inertial measurements to estimate the 6D pose of the sensor. It is based on tightly-coupled sensor fusion of the UWB and the inertial sensors. We formulate the sensor fusion problem as a MAP problem (2), estimating the state vector

$x_{1: N}=\left\{\left\{p_{t}^{\mathrm{n}}, v_{t}^{\mathrm{n}}, q_{t}^{\mathrm{nb}}\right\}_{t=1}^{N},\left\{\tau_{k}\right\}_{k=1}^{K} \delta_{\mathrm{a}}, \delta_{\omega}, \sigma_{\mathrm{a}}, \sigma_{\omega}, \sigma, \gamma\right\}$,

where $p_{t}^{\mathrm{n}}$ and $v_{t}^{\mathrm{n}}$ denote the sensor position and velocity at time $t$, respectively. Both of these are expressed in the navigation frame $n$. The sensor orientation is denoted $q_{t}^{\mathrm{nb}}$. Note that we encode the orientation state using a three-dimensional state vector around a linearization point represented by a unit quaternion [6], [27]-[29]. The UWB system typically obtains measurements at a lower frequency than the inertial measurement frequency. Hence, we use a subscript $k$ to denote the UWB measurements while using a subscript $t$ to denote the inertial measurements. The state $x_{1: N}$ is modeled for each time $t$.

In our approach, we make use of a dynamic model where the inertial measurements can be thought of as inputs. Hence, we model the position, the velocity and the orientation of the IMU in terms of the sensor acceleration and angular velocity as

$$
\begin{aligned}
p_{t+1}^{\mathrm{n}} & =p_{t}^{\mathrm{n}}+T v_{t}^{\mathrm{n}}+\frac{T^{2}}{2} a_{t}^{\mathrm{n}} \\
& =p_{t}^{\mathrm{n}}+T v_{t}^{\mathrm{n}}+\frac{T^{2}}{2} R_{t}^{\mathrm{nb}}\left(y_{\mathrm{a}, t}-\delta_{\mathrm{a}}-e_{\mathrm{a}, t}\right)+\frac{T^{2}}{2} g^{\mathrm{n}}, \\
v_{t+1}^{\mathrm{n}} & =v_{t}^{\mathrm{n}}+T a_{t}^{\mathrm{n}} \\
& =v_{t}^{\mathrm{n}}+T R_{t}^{\mathrm{nb}}\left(y_{\mathrm{a}, t}-\delta_{\mathrm{a}}-e_{\mathrm{a}, t}\right)+T g^{\mathrm{n}}, \\
q_{t+1}^{\mathrm{nb}} & =q_{t}^{\mathrm{nb}} \odot \exp \left(\frac{T}{2} \omega_{t}\right),
\end{aligned}
$$

where $T$ denotes the IMU sampling interval. The acceleration $a_{t}^{\mathrm{n}}$ is obtained from 9 . The orientation $q_{t}^{\mathrm{nb}}$ is modeled in terms of the angular velocity $\omega_{t}$ obtained from (8). In 19c, $\odot$ denotes the quaternion product and exp denotes the vector exponential

$$
\exp \left(\frac{T}{2} \omega_{t}\right)=\left(\cos \left\|\frac{T}{2} \omega_{t}\right\|_{2} \quad \frac{\omega_{t}^{\top}}{\left\|\omega_{t}\right\|_{2}} \sin \left\|\frac{T}{2} \omega_{t}\right\|_{2}\right)^{\top}
$$

For more details on quaternion algebra, see e.g. [6], [30]. Note that we interchangeably make use of the unit quaternion $q^{\mathrm{nb}}$ and the rotation matrix $R^{\mathrm{nb}}$ as representations of the orientation. Furthermore, we use the notation $R^{\mathrm{bn}}=\left(R^{\mathrm{nb}}\right)^{\top}$ for the inverse rotation.

In the measurement model, the UWB measurements, modeled as in (3), are used to update the state. For this, the IMU and the UWB transmitter are assumed to be rigidly attached to each other. The position of the transmitter with respect to the IMU is assumed to be known.

The state is computed using the following MAP problem

$$
\begin{aligned}
& \widehat{x}_{1: N}^{\mathrm{MAP}}=\underset{x_{1: N}}{\arg \min }-\sum_{t=1}^{N} \log p\left(x_{t+1} \mid x_{t}, y_{\mathrm{a}, t}, y_{\omega, t}\right)- \\
& \sum_{k=1}^{K} \sum_{m=1}^{M} \log p\left(y_{\mathrm{u}, m k} \mid x_{t(k)}\right)
\end{aligned}
$$

where the first term denotes the dynamic model described by (19). The second term denotes the measurement model, using the UWB measurement model (3), the asymmetric heavy-tailed distribution (4) and the relative position of the transmitter with respect to the IMU. The problem (21) can be solved ${ }^{2}$ using a standard Gauss-Newton algorithm, see e.g. [25], [26]. Since the sensor fusion problem is nonlinear, parts of the problem are solved first to provide good initial estimates. The resulting pose estimation approach is summarized in Algorithm 2 .

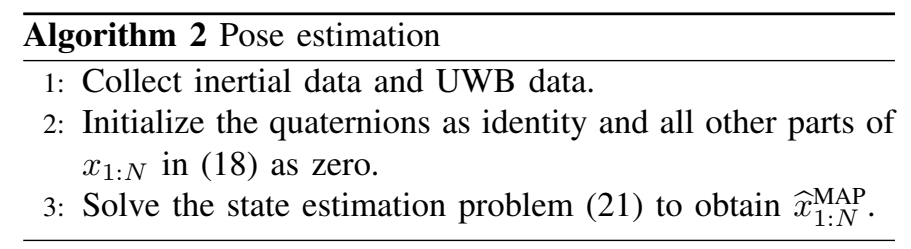

\section{EXPERIMENTAL RESULTS}

In our experiments, we use an UWB setup consisting of 10 receivers deployed in a room with a size of approximately $8 \times 6 \times 2.5 \mathrm{~m}$. A test-subject walks around the measurement volume along a circular path for approximately $24 \mathrm{~s}$. An IMU and an UWB transmitter have been attached to his foot. The UWB measurements are significantly affected by the NLOS conditions and multipath since the transmitter is quite close to the ground and since the body frequently blocks the direct path to the receivers. Optical markers have also been placed on the body to provide reference data. These optical markers are tracked by a camera system [31]. This industry-standard system has an accuracy that is an order of magnitude larger than the accuracy expected from our UWB system. Hence (using multiple markers), the camera system can provide both ground truth position and orientation estimates.

In Section VII-A we will use the UWB data from the transmitter on the subject's foot to experimentally validate the proposed asymmetric distribution (4). We will show that using this distribution in the multilateration approach described in Section V-B, considerably better position estimates are obtained than when the same approach would be used assuming Gaussian or Cauchy distributed noise. In Section VII-B we will present our calibration results. In Section VII-C we will present our sensor fusion results.

\section{A. Experimental validation of the asymmetric noise distribu- tion}

In this section we will use the multilateration approach introduced in Section V-B while assuming that the system has previously been calibrated. We will use three different assumptions on the noise used in (16), namely

1) the asymmetric noise distribution (4) with parameters $\theta_{\mathrm{A}}=\left(\left\{t_{k}^{\mathrm{n}}, \tau_{k}\right\}_{k=1}^{K}, \sigma, \gamma\right)$

\footnotetext{
${ }^{2}$ Also for the sensor fusion problem, good initial estimates help for convergence. Hence, we first estimate only a part of the state vector (18), choosing the parameters related to the noise characteristics as a fixed value. We also use a Gaussian prior for the sensor biases. The resulting parameters are then used to determine a first estimate of $\sigma_{\mathrm{a}}, \sigma_{\omega}, \sigma$ and $\gamma$. Finally, the obtained estimates are used as initial values for the final optimization 21.
} 
TABLE I

RMSE FOR THE POSITION USING THE MULTILATERATION APPROACH INTRODUCED IN SECTION V-B AS COMPARED TO DATA FROM AN OPTICAL REFERENCE SYSTEM ASSUMING THE NOISE TO BE DISTRIBUTED ACCORDING TO THE ASYMMETRIC DISTRIBUTION (4), ACCORDING TO A CAUCHY DISTRIBUTION AND ACCORDING TO A GAUSSIAN DISTRIBUTION.

\begin{tabular}{lrrr}
\hline & $\mathrm{x}[\mathrm{cm}]$ & $\mathrm{y}[\mathrm{cm}]$ & $\mathrm{z}[\mathrm{cm}]$ \\
\hline Asymmetric distribution 4 & 5.9 & 7.2 & 12.2 \\
Cauchy distribution & $22.0^{*}$ & $9.2^{*}$ & $19.1^{*}$ \\
Gaussian distribution & $53.4^{*}$ & $75.9^{*}$ & $176.9^{*}$ \\
\hline
\end{tabular}

* One measurement has been discarded in computing these RMSE values. These measurements arise around $11 \mathrm{~s}$ for the Cauchy distribution and around $22 \mathrm{~s}$ for the Gaussian distribution and deviate from the reference positions by more than $100 \mathrm{~m}$.

2) a Cauchy distribution with parameters $\theta_{\mathrm{C}}=\left(\left\{t_{k}^{\mathrm{n}}, \tau_{k}\right\}_{k=1}^{K}, \gamma\right)$

3) a Gaussian distribution with parameters $\theta_{\mathrm{G}}=$ $\left(\left\{t_{k}^{\mathrm{n}}, \tau_{k}\right\}_{k=1}^{K}, \sigma\right)$.

The resulting position estimates are shown in Fig. 7. The dashed lines are the position estimates from the optical reference system. As can be seen, the position estimates are best for the asymmetric distribution and worst for the Gaussian distribution. This is also summarized in Table 1 in terms of the root mean square error (RMSE) for the position. In Fig. 8 the residuals including their estimated PDFs are plotted for all three cases. As can be seen, the Gaussian is clearly not a good fit due to the large number of outliers. Although the estimated Cauchy distribution seems to describe the residuals reasonably well, this model allows for physically unreasonable negative residuals, i.e. pulses traveling faster than the speed of light. Hence, also from these histograms it can be concluded that the asymmetric noise distribution (4) offers the best model for the experimental data. Note that these results are highly dependent on the number of outliers in the UWB data. On "cleaner" UWB data, the difference in position accuracy between the different distributions would of course be less.

\section{B. Calibration}

Algorithm 1 has been used to compute an estimate of the positions and clock offsets of the receivers in the UWB setup. The estimated trajectory of the transmitter and the ML estimates of the receiver positions are depicted in Fig. 9. The smoothness of the estimated transmitter trajectory suggests that good multilateration results are obtained and hence gives confidence also in the resulting calibration results. Fig. 10 shows a histogram of the residuals from the calibration algorithm. As can be seen, the estimated PDF (shown in red) fits the data reasonably well. Furthermore, the calibration has been used in the sensor fusion algorithm with good results (as will be discussed in the subsequent section), which indirectly validates the quality of the calibration.

The results described in this section have been obtained using an inefficient implementation of Algorithm 1 in Matlab. However, efficient implementation should be possible due to the sparsity inherent in the problem and the typical problem

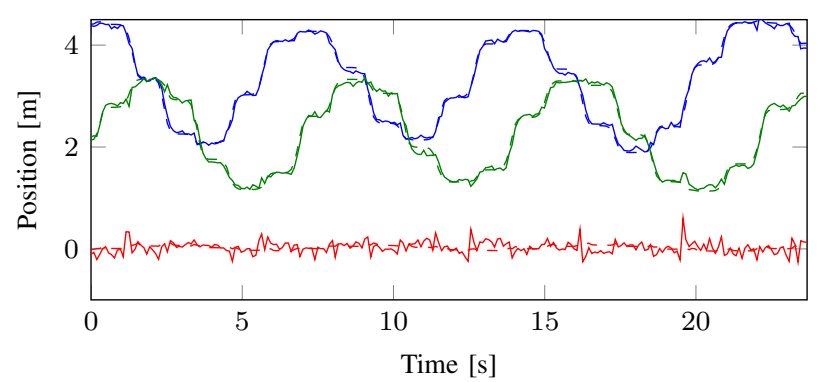

(a) Asymmetric

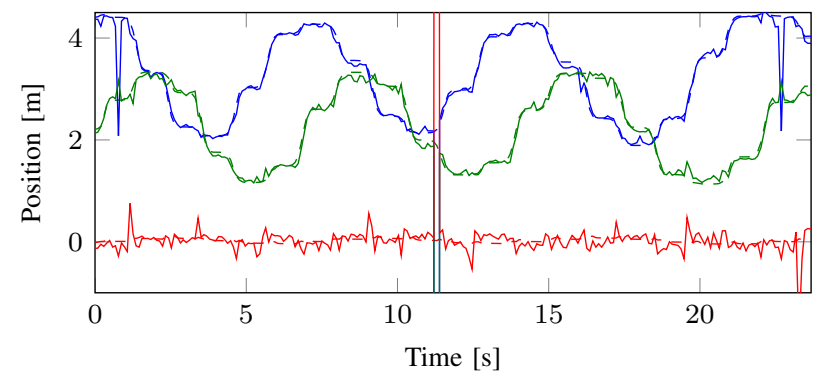

(b) Cauchy

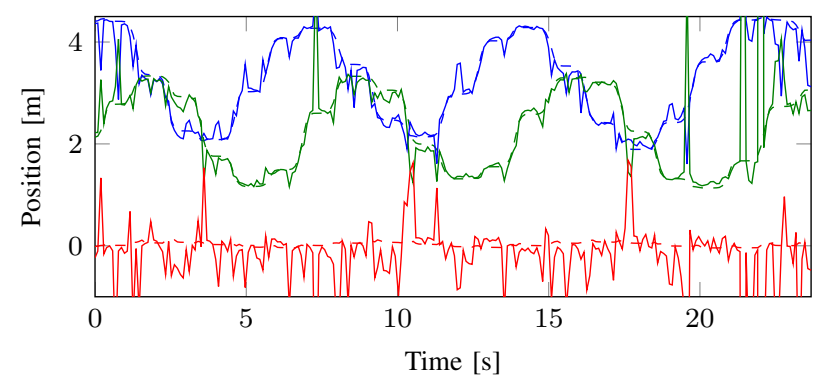

(c) Gaussian

Fig. 7. Position estimates from the multilateration approach 16 using the measurements from the transmitter on the foot. The estimated positions using the asymmetric heavy-tailed noise distribution (4) are depicted in (a). The different colors denote the different directions with $\mathrm{x}$ in blue, $\mathrm{y}$ in green and $\mathrm{z}$ in red. The estimated positions using a Cauchy and a Gaussian noise distribution are depicted in (b) and (c), respectively. Data from an optical reference system is included as dashed lines in each plot for comparison.

dimensions of less than 2500 parameters (this corresponds to collecting calibration data $\mathcal{D}_{1}$ for one minute at $10 \mathrm{~Hz}$ ). The sparsity pattern of the matrix that needs inversion in the constrained Gauss-Newton algorithm, consisting of the approximate Hessian and the gradients of the constraints (see e.g. [25]), is shown in Fig. 11. Note that since our calibration problem is nonlinear, this matrix inversion needs to be performed several times.

\section{Pose estimation}

To evaluate the proposed pose estimation solution (Algorithm 2), it has been used to track the motion of an IMU and an UWB transmitter placed on the foot of a test-subject walking in an indoor environment, using the experiment already introduced in Section VII-A. The IMU provides $120 \mathrm{~Hz}$ inertial measurements. The UWB pulses are transmitted at $10 \mathrm{~Hz}$.

Fig. 12 shows an overview of the position estimated using Algorithm 2. The positions of the UWB receivers are shown in 


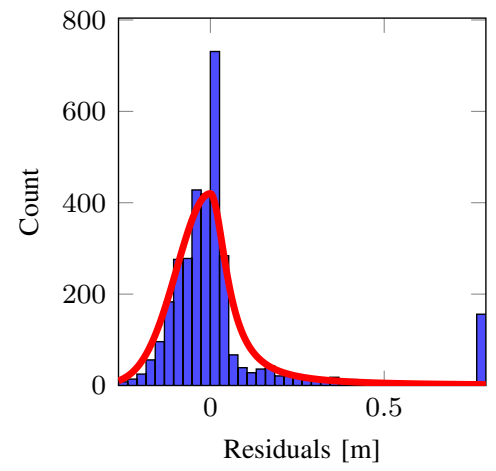

(a) Asymmetric

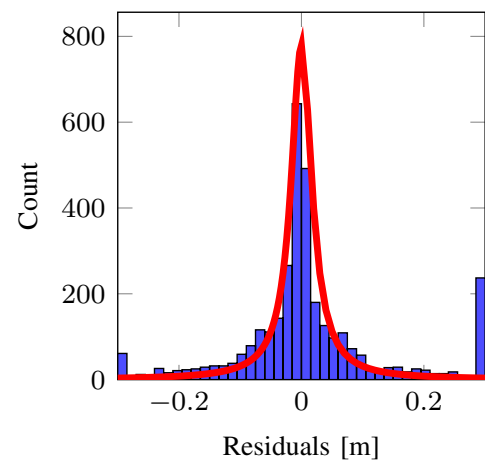

(b) Cauchy

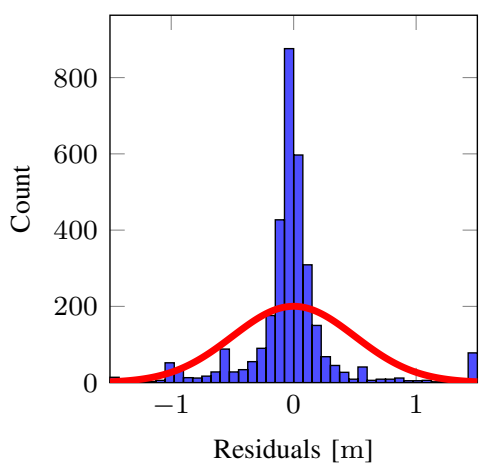

(c) Gaussian

Fig. 8. Residuals from the multilateration approach including their estimated PDFs using (a) the asymmetric distribution (4), a symmetric Cauchy distribution (b) and a symmetric Gaussian distribution (c). The residuals outside of the scope of the figures have been collected in the outermost bins. Note the different scales on the $x$-axes and the fact that the left plot is not centered around 0 to emphasize the asymmetric nature of this distribution. The quantities in the measurement equation (3) are all expressed in meters resulting in residuals in meters.

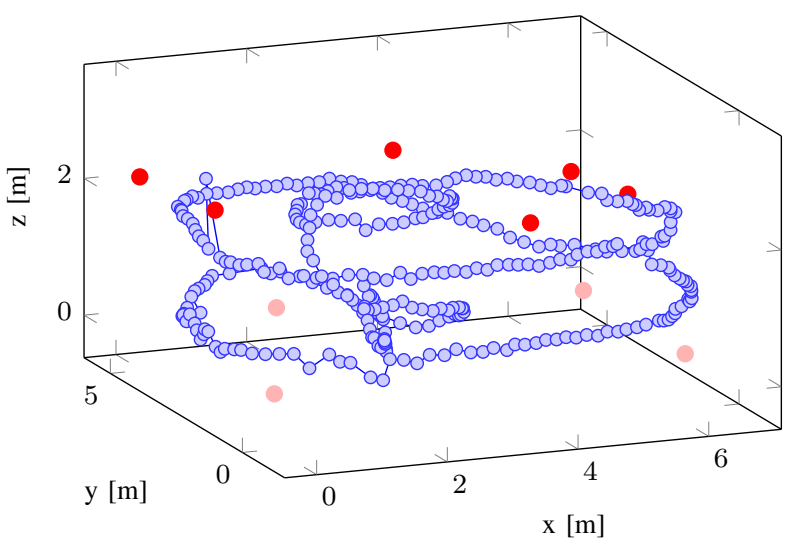

Fig. 9. Calibration results of the UWB setup. The estimated transmitter positions are depicted in blue. The receivers in our UWB setup are either placed close to the ground or close to the ceiling. The positions of the receivers close to the ceiling are depicted in bright red and the positions of the receivers close to the ground are depicted in light red.

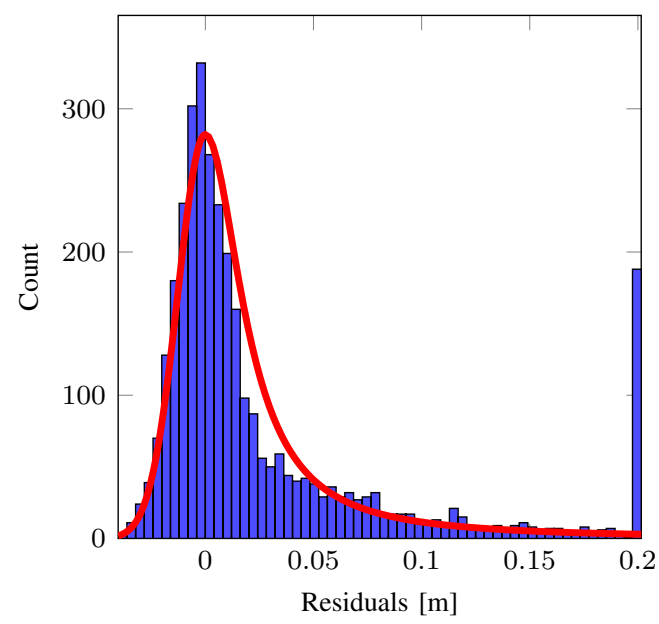

Fig. 10. Residuals from the calibration algorithm and the estimated PDF. Note that the residuals outside of the scope of the figure have been collected in the outermost bins and that the plot is not centered around 0 to emphasize the asymmetric nature of the distribution. The quantities in the measurement equation (3) are all expressed in meters resulting in residuals in meters.

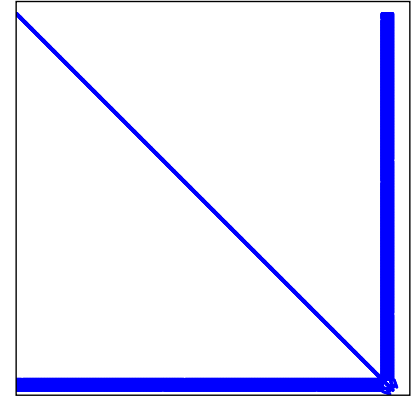

Fig. 11. The sparsity pattern of the matrix that needs inversion in the constrained Gauss-Newton algorithm, consisting of the approximate Hessian and the gradients of the constraints. The block-diagonal part is due to the independency of the different UWB pulses. The arrow-point is due to the model dependency of each pulse on the receiver positions and their clock offsets (see (3). Only $5.7 \%$ of the matrix elements is non-zero.

red. The circular path is clearly recognizable. It only occupies a small part of the measurement volume of the UWB tracking system so that a performance comparison with an optical reference system is possible.

Figs. 13 and 14 show the estimated position and orientation as compared to those from the optical reference system. It can be concluded that our solution is capable of producing a driftfree and accurate pose estimate at a high output frequency. In fact, the comparison shows $3 \mathrm{~cm}$ RMSE for position and less than $1^{\circ}$ RMSE for orientation, see Table III.

As for the UWB calibration algorithm, our implementation has not been optimized in terms of computational speed. However, the sensor fusion problem is inherently sparse due to the Markov property of the state and can hence be solved efficiently.

\section{CONCLUSIONS AND FUTURE WORK}

We have presented a sensor fusion approach to combine inertial measurements with TOA measurements from an UWB system for $6 \mathrm{D}$ pose estimation. The approach is experimentally shown to result in accurate position and orientation estimates when compared to data from an independent optical reference system. To be able to use the UWB measurements in the sensor 


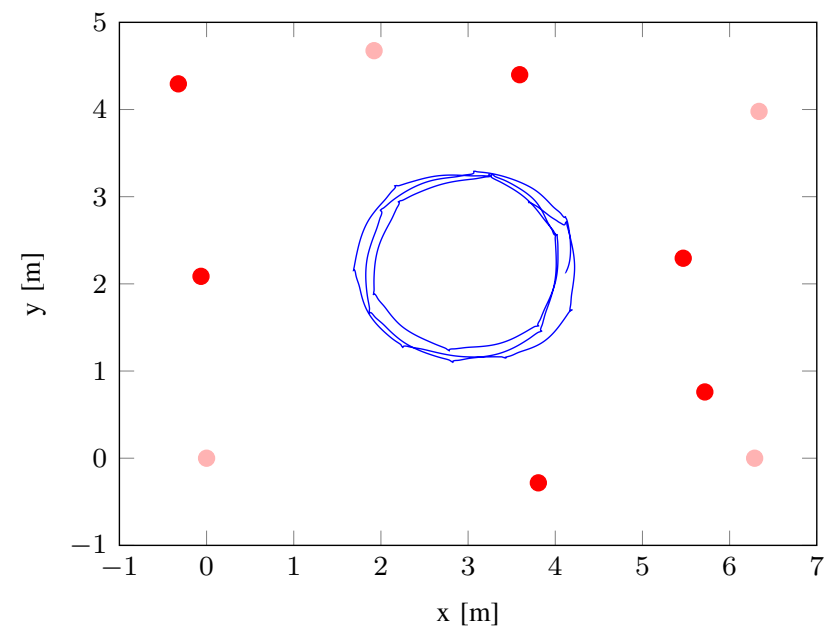

Fig. 12. Top view of the experiment where the subject walked along a circular path. The estimated trajectory $p_{1: N}^{\mathrm{n}}$ of the IMU on the subject's foot is shown in blue. The positions of the receivers close to the ceiling are depicted in bright red. The positions of the receivers close to the floor are depicted in light red.
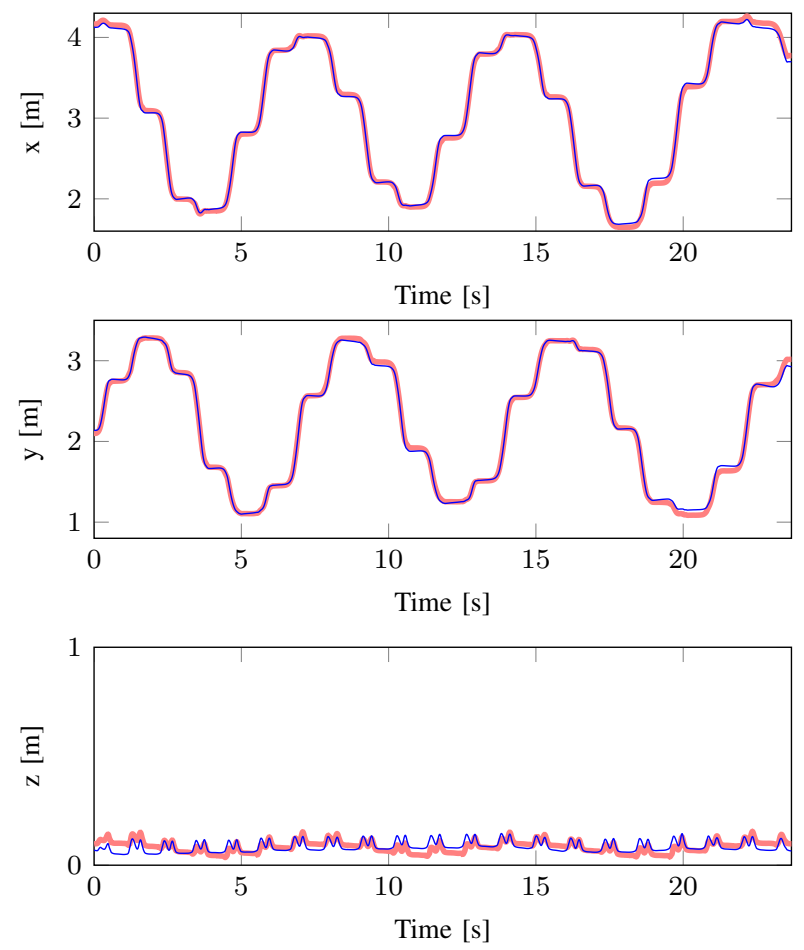

Fig. 13. Position of the IMU $p_{1: N}^{\mathrm{n}}$ on the subject's foot. The estimates from Algorithm 2 are depicted in blue. The estimates from the optical reference system are depicted in thick red.

TABLE II

RMSE FOR THE POSITION AND ORIENTATION ESTIMATES FROM ALGORITHM 2 AS COMPARED TO DATA FROM THE OPTICAL REFERENCE SYSTEM.

\begin{tabular}{lccc}
\hline & $\mathrm{x}$ & $\mathrm{y}$ & $\mathrm{z}$ \\
\hline position $[\mathrm{cm}]$ & 3.0 & 3.0 & 2.3 \\
orientation $\left[{ }^{\circ}\right]$ & 0.37 & 0.44 & 0.69 \\
\hline
\end{tabular}
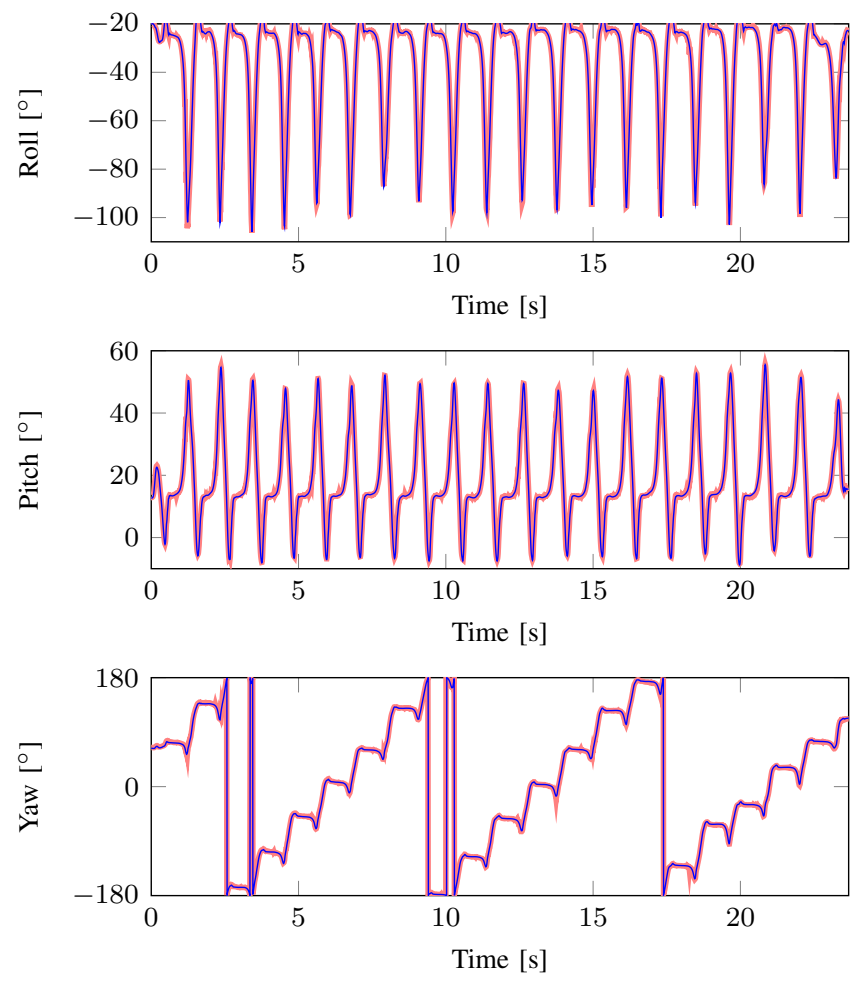

Fig. 14. Orientation of the IMU $q_{1: N}^{\text {nb }}$ on the subject's foot expressed using Euler angles (roll, pitch, yaw). The estimates from Algorithm 2 are depicted in blue. The estimates from the optical reference system are depicted in thick red.

fusion approach, the UWB setup has to be calibrated, i.e. the receiver positions and their clocks offsets have to be computed. We have solved the UWB calibration problem using a novel approach, taking into account the possibility of delayed UWB measurements due to NLOS and/or multipath. Throughout this work, we have used an asymmetric heavy-tailed distribution to model the outliers in the UWB measurements. This model is shown to lead to accurate position estimates even from challenging data containing a fairly large amount of outliers in a new multilateration approach.

An interesting direction for future work is to combine the sensor fusion algorithm introduced in this work with the motion capture approach in [8]. This would open up for the possibility of combining information from multiple IMUs and multiple UWB transmitters to determine the pose of multiple body segments or even the entire human body or any other objects with multiple connected parts.

\section{ACKNOWLEDGEMENTS}

This work is supported by CADICS, a Linnaeus Center funded by the Swedish Research Council (VR), by BALANCE, a European Commission, FP7 research project and by the project Probabilistic modeling of dynamical systems (Contract number: 621-2013-5524) funded by the Swedish Research Council (VR).

\section{REFERENCES}

[1] Ubisense, http://www.ubisense.net Accessed on April 8, 2014. 
[2] Time Domain, http://www.timedomain.com Accessed on April 7, 2014.

[3] S. Gezici, Z. Tian, G. B. Giannakis, H. Kobayashi, A. F. Molisch, H. V. Poor, and Z. Sahinoglu, "Localization via ultra-wideband radios: a look at positioning aspects for future sensor networks," IEEE Signal Processing Magazine, vol. 22, no. 4, pp. 70-84, 2005.

[4] Xsens Technologies B.V., http://www.xsens.com Accessed on April 8, 2014.

[5] G. Bellusci, D. Roetenberg, F. Dijkstra, H. J. Luinge, and P. Slycke, "Xsens MVN MotionGrid: Drift-free human motion tracking using tightly coupled ultra-wideband and miniature inertial sensors," June 2011.

[6] J. D. Hol, "Sensor fusion and calibration of inertial sensors, vision, ultrawideband and GPS," Ph.D. dissertation, Linköping University, Sweden, 2011.

[7] J. D. Hol, F. Dijkstra, H. Luinge, and T. B. Schön, “Tightly coupled UWB/IMU pose estimation," in Proceedings of the IEEE International Conference on Ultra-Wideband (ICUWB), Vancouver, Canada, September 2009, pp. 688-692.

[8] M. Kok, J. D. Hol, and T. B. Schön, "An optimization-based approach to human body motion capture using inertial sensors," in Proceedings of the 19th World Congress of the International Federation of Automatic Control, Cape Town, South Africa, August 2014.

[9] J. D. Hol, T. B. Schön, and F. Gustafsson, "Ultra-wideband calibration for indoor positioning," in Proceedings of the IEEE International Conference on Ultra-Wideband (ICUWB), Nanjing, China, September 2010, pp. 1-4.

[10] Z. Sahinoglu, S. Gezici, and I. Güvenc, Ultra-wideband Positioning Systems: Theoretical Limits, Ranging Algorithms, and Protocols. Cambridge University Press, 2008.

[11] Y. T. Chan and K. C. Ho, "A simple and efficient estimator for hyperbolic location," IEEE Transactions on Signal Processing, vol. 42, no. 8, pp. 1905-1915, 1994.

[12] A. H. Sayed, A. Tarighat, and N. Khajehnouri, "Network-based wireless location: challenges faced in developing techniques for accurate wireless location information," IEEE Signal Processing Magazine, vol. 22, no. 4, pp. 24-40, 2005.

[13] F. Gustafsson, Statistical Sensor Fusion. Studentlitteratur, 2012.

[14] A. M. Zoubir, V. Koivunen, Y. Chakhchoukh, and M. Muma, "Robust estimation in signal processing: A tutorial-style treatment of fundamental concepts," IEEE Signal Processing Magazine, vol. 29, no. 4, pp. 61-80, 2012.

[15] N. A. Alsindi, B. Alavi, and K. Pahlavan, "Measurement and modeling of ultrawideband TOA-based ranging in indoor multipath environments," IEEE Transactions on Vehicular Technology, vol. 58, no. 3, pp. 10461058, 2009.

[16] F. Gustafsson and F. Gunnarsson, "Mobile positioning using wireless networks: possibilities and fundamental limitations based on available wireless network measurements," IEEE Signal Processing Magazine, vol. 22, no. 4, pp. 41-53, 2005.

[17] A. Prorok and A. Martinoli, "Accurate indoor localization with ultrawideband using spatial models and collaboration," The International Journal of Robotics Research, vol. 33, no. 4, pp. 547-568, 2014.

[18] N. Patwari, J. N. Ash, S. Kyperountas, A. O. Hero, R. L. Moses, and N. S. Correal, "Locating the nodes: cooperative localization in wireless sensor networks," IEEE Signal Processing Magazine, vol. 22, no. 4, pp. 54-69, 2005.

[19] S. Sczyslo, J. Schroeder, S. Galler, and T. Kaiser, "Hybrid localization using UWB and inertial sensors," in Proceedings of the IEEE International Conference on Ultra-Wideband (ICUWB), vol. 3, Hannover, Germany, September 2008, pp. 89-92.

[20] S. Pittet, V. Renaudin, B. Merminod, and M. Kasser, "UWB and MEMS based indoor navigation," Journal of Navigation, vol. 61, no. 3, pp. 369384, July 2008.

[21] J. A. Corrales, F. A. Candelas, and F. Torres, "Hybrid tracking of human operators using IMU/UWB data fusion by a Kalman filter," in Proceedings of the 3rd ACM/IEEE International Conference on HumanRobot Interaction (HRI), 2008, pp. 193-200.

[22] A. De Angelis, J. Nilsson, I. Skog, P. Händel, and P. Carbone, "Indoor positioning by ultrawide band radio aided inertial navigation," Metrology and Measurement Systems, vol. 17, no. 3, pp. 447-460, 2010.

[23] L. Zwirello, X. Li, T. Zwick, C. Ascher, S. Werling, and G. F. Trommer, "Sensor data fusion in UWB-supported inertial navigation systems for indoor navigation," in Proceedings of the IEEE International Conference on Robotics and Automation (ICRA), Karlsruhe, Germany, 2013, pp. 3154-3159.

[24] U. Hammes, E. Wolsztynski, and A. M. Zoubir, "Robust tracking and geolocation for wireless networks in NLOS environments," IEEE
Journal of Selected Topics in Signal Processing, vol. 3, no. 5, pp. 889901, 2009.

[25] S. Boyd and L. Vandenberghe, Convex Optimization. Cambridge University Press, 2004.

[26] J. Nocedal and S. J. Wright, Numerical Optimization, 2nd ed. Springer Series in Operations Research, 2006.

[27] M. Kok, Probabilistic modeling for positioning applications using inertial sensors. Licentiate thesis, Linköping University, Sweden, 2014.

[28] J. L. Crassidis, F. L. Markley, and Y. Cheng, "A survey of nonlinear attitude estimation methods," Journal of Guidance, Control, and Dynamics, vol. 30, no. 1, pp. 12-28, 2007.

[29] G. Grisetti, R. Kummerle, C. Stachniss, U. Frese, and C. Hertzberg, "Hierarchical optimization on manifolds for online 2D and 3D mapping," in Proceedings of the IEEE International Conference on Robotics and Automation (ICRA), Anchorage, Alaska, 2010, pp. 273-278.

[30] J. B. Kuipers, Quaternions and Rotation Sequences: a primer with applications to orbits, aerospace, and virtual reality. Princeton University Press, 1999.

[31] Vicon, http://www.vicon.com Accessed on October 6, 2014.

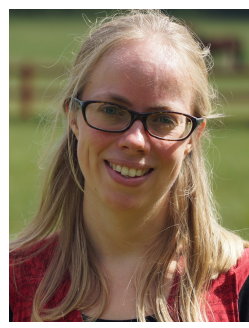

Manon Kok is a Ph.D. student in Automatic Control at the Department of Electrical Engineering at Linköping University. She received a M.Sc. degree in Applied Physics in Jan. 2009 and a M.Sc. degree in Philosophy of Science, Technology and Society in June 2007, both from the University of Twente, the Netherlands. Between Feb. 2009 and July 2011 she worked as a research engineer at Xsens Technologies B.V., the Netherlands. Her main research interests are in probabilistic modeling for position and orientation estimation using inertial sensors.

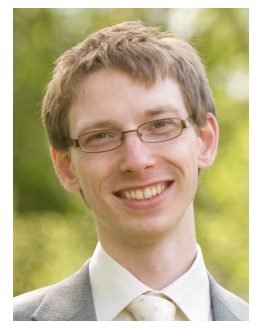

Jeroen D. Hol is a senior research engineer at Xsens Technologies B.V., the Netherlands. He received the Ph.D. degree in Automatic Control in June 2011 from Linköping Universty, Sweden, and the M.Sc. degree (cum laude) in Mechanical Engineering in 2005 from the University of Twente, The Netherlands. His main research topics are sensor fusion and calibration for inertial sensors, motion capture and ultrawideband.

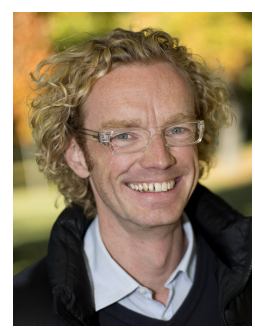

Thomas B. Schön is Professor of the Chair of Automatic Control in the Department of Information Technology at Uppsala University. He received the $\mathrm{PhD}$ degree in Automatic Control in Feb. 2006, the MSc degree in Applied Physics and Electrical Engineering in Sep. 2001, the BSc degree in Business Administration and Economics in Jan. 2001, all from Linköping University. He has held visiting positions with the University of Cambridge (UK), the University of Newcastle (Australia) and Universidad Técnica Federico Santa María (Valparaíso, Chile). $\mathrm{He}$ is a Senior member of the IEEE. He was awarded the Automatica best paper price in 2014 and in 2013 he received the best $\mathrm{PhD}$ thesis award by The European Association for Signal Processing (EURASIP). He received the best teacher award at the Institute of Technology, Linköping University in 2009. Schön's main research interest is nonlinear inference problems, especially within the context of dynamical systems, solved using probabilistic methods. 